SPACE [1] and beamline control software BSS [2]. For smooth communication with distant users via the Internet, we have newly developed the data management system DCha (Database for Crystallography with Home-lab Arrangements) which mediates between the users and SPring- 8 beamlines. D-Cha provides the GUI for users to deposit the experimental conditions for samples and to browse / download the collected data on web browser.

The mail-in system has been developed and operated for Structural Genomics Project at RIKEN Structural Genomics II (BL26B2) since September 2005. Then the system has been presented for public users at Structural Biology III (BL38B1) since December 2005 and at RIKEN Structural Genomics I (BL26B1) since December 2006. At BL26B1 and BL26B2, mail-in system has been operated on a daily basis. In addition, the commercial mail-in service has just started in July 2006, as the joint project among JASRI, RIKEN and analysis service companies. The mail-in data collection is our first step of remote beamline access at SPring- 8 . The next step is to achieve the fully remote control data collection based on the mail-in system.

[1] Ueno G, Hirose R, Ida K, Kumasaka T, Yamamoto M, J Appl. Cryst. 2004 Dec;37(Pt 6):867-873 "Sample management system for a vast amount of frozen crystals at SPring-8"

[2] Ueno G, Kanda H, Kumasaka T, Yamamoto M, J Synchrotron Radiat. 2005 May;12(Pt 3):380-4 "Beamline Scheduling Software: administration software for automatic operation of the RIKEN structural genomics beamlines at SPring-8"

\section{MS02 P05}

Recent advances in the Crank automated structure solution suite Navraj S. Pannu, Pavol Skubak, Irakli Sikharulidze, Jan Pieter Abrahams, Rudolf A.G. de Graaff. Biophysical Structural Chemistry, Leiden University, Leiden, The Netherlands.

E-mail: raj@.chem.leidenuniv.nl

Keywords: automated structure solution, MAD, SAD For its first release, the CRANK system was shown to effectively detect anomalous scatterers and phase SAD data [1]. Since then, CRANK's speed and robustness has improved, building many structures automatically for SAD, SIRAS, MAD and MAD + native data [2].

One improvement involves using Luzzati parameters refined in the program BP3 to validate the quality and completeness of a substructure obtained. This has proven to be effective and reliable in identifying correct solutions that do not meet the figure of merit levels reported in substructure detection programs necessary to safely assume that a correct solution was found. Thus, this allows for an early termination of substructure detection. To improve automated model building, an interface to ARP/wARP and REFMAC has been added to also include SAD data directly in model refinement. The multivariate SAD likelihood function, implemented in a modified version of REFMAC has been shown to extend the resolution and phase quality limits required for automated model building with iterative refinement [3]. Recently, the new refinement target was shown to be very effective in combination with the SHELX[C/D/E $]$ pipeline available in CRANK.

The above and other advances are in the latest version of CRANK at http://www.bfsc.leidenuniv.nl/software/crank or the CCP4 pre-release zone.

[1] Ness, S, de Graaff RA, Abrahams, JP, Pannu NS. (2004) Structure, 12, 1753. [2]http://www.bfsc.leidenuniv.nl/software/crank/tests/pipeline1.ht $\mathrm{ml}$

[3] Skubak, P, Ness, S, Pannu, NS. Acta Cryst D61, 1626.

\author{
MS02 P06 \\ Truncate2 - A Program for Intensity to Amplitude \\ Conversion Norman Stein ${ }^{\mathrm{a}}$, Charles Ballard ${ }^{\mathrm{a}},{ }^{\mathrm{a}} C C P 4$, \\ Daresbury Laboratory, Warrington, WA4 4AD, UK. \\ E-mail: n.d.stein@dl.ac.uk
}

Keywords: software, protein structure function, structure solution

Conversion of measured intensities to structure factors in protein crystallography is complicated by the fact that background subtraction can result in negative intensities for weak reflections. Truncate 2 is a new CCP4 program, designed to replace the original Truncate program, which uses Bayesian statistics to produce positive structure factor values from negative input intensities. Small positive intensities are also boosted by the conversion process. In addition, Truncate 2 calculates a number of statistics from the intensity data, such as moments, cumulative intensity distributions and the Wilson plot. When output in graphical form, these can be used to assess data quality and to check for possible twinning. Truncate2 detects significant anisotropy in the data and performs anisotropy correction. A number of quantitative tests for twinning such as the $\mathrm{H}$ test and the Britton test have also been introduced. The prior distribution used in Truncate is the Wilson distribution, which is only appropriate in the absence of twinning and translational NCS. Truncate2 is capable of handling the last two cases in a more accurate manner.

\section{MS02 P07}

New developments for a full automation of the FIP beamline at the ESRF Jacquamet L., Bertoni A., Borel F., Charrault P., Israel-Gouy P., Iwema T., Kahn R., Joly J., Ohana J., Pirocchi M., Robin A., Serre L., Vernede X. and Ferrer J. L. Institut de Biologie Structurale Jean Pierre Ebel, CEA; CNRS; Université Joseph Fourier; 41 rue Jules Horowitz,F-38027 Grenoble, France .

E-mail: lilian.jacquamet@ibs.fr

Keywords: protein data collection, automatic control, robots

FIP (French beamline for the Investigation of Proteins) at the ESRF (European Synchrotron Radiation Facility) pushed developments in automation to reach a fully automated beamline.

- The energy adjustment and beam optimization are completely automated [1].

- The screening of the different protein crystals is ensured by a robotic system: CATS (Cryogenic Automated Transfer System) [2] now commercialized by IRELEC.

- In addition, this robot offers the possibility to analyze crystals directly as they grow in drops inside crystallization plates [3]. FIP has then developed in collaboration with GREINER Bio-One a new crystallization plate devoted to this new application.

- The centering of the protein crystal is improved using the installed UV-laser [4].

- The automation of the data recording and processing with ADP (Automated Data Processing) [5] has also been achieved. 